\title{
Hydroxyl radical reactivity at the air-ice interface
}

\author{
T. F. Kahan ${ }^{1}$, R. Zhao ${ }^{1}$, and D. J. Donaldson ${ }^{1,2}$ \\ ${ }^{1}$ Department of Chemistry, University of Toronto, 80 Saint George Street, Toronto, M5S 3H6 Ontario, Canada \\ ${ }^{2}$ Department of Physical and Environmental Sciences, University of Toronto, Scarborough, Canada
}

Received: 14 September 2009 - Published in Atmos. Chem. Phys. Discuss.: 5 October 2009

Revised: 2 January 2010 - Accepted: 8 January 2010 - Published: 26 January 2010

\begin{abstract}
Hydroxyl radicals are important oxidants in the atmosphere and in natural waters. They are also expected to be important in snow and ice, but their reactivity has not been widely studied in frozen aqueous solution. We have developed a spectroscopic probe to monitor the formation and reactions of hydroxyl radicals in situ. Hydroxyl radicals are produced in aqueous solution via the photolysis of nitrite, nitrate, and hydrogen peroxide, and react rapidly with benzene to form phenol. Similar phenol formation rates were observed in aqueous solution and bulk ice. However, no reaction was observed at air-ice interfaces, or when bulk ice samples were crushed prior to photolysis to increase their surface area. We also monitored the heterogeneous reaction between benzene present at air-water and air-ice interfaces with gas-phase $\mathrm{OH}$ produced from HONO photolysis. Rapid phenol formation was observed on water surfaces, but no reaction was observed at the surface of ice. Under the same conditions, we observed rapid loss of the polycyclic aromatic hydrocarbon $(\mathrm{PAH})$ anthracene at air-water interfaces, but no loss was observed at air-ice interfaces. Our results suggest that the reactivity of hydroxyl radicals toward aromatic organics is similar in bulk ice samples and in aqueous solution, but is significantly suppressed in the quasi-liquid layer (QLL) that exists at air-ice interfaces.
\end{abstract}

\section{Introduction}

Reactions involving hydroxyl radicals are of great interest in environmental chemistry. Hydroxyl radicals react readily with organic pollutants in the atmosphere and in natural waters; it is expected that the same is true in snow and ice (e.g. Anastasio et al., 2007). If they occur, such reac-

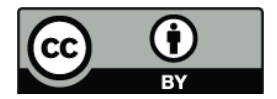

Correspondence to: D. J. Donaldson (jdonalds@chem.utoronto.ca) tions would be important both in snow-covered urban centres, where organic pollutants are ubiquitous, and also in remote polar regions, where organic pollutants such as polycyclic aromatic hydrocarbons (PAHs) have been detected in snow and ice (Schrimpff et al., 1979; Jaffrezo et al., 1994; Franz and Eisenreich, 2000; Masclet et al., 2000; Carrera et al., 2001; Compoint et al., 2002; Slater et al., 2002; Fernandez et al., 2003; Melnikov et al., 2003; Cincinelli et al., 2005; Daly and Wania, 2005; Herbert et al., 2006; Wang et al., 2008a, b). Hydroxyl radical mixing ratios in the air above polar snowpacks can be as high as those in urban centres, due primarily to photochemical production within the snowpack. (See (Domine and Shepson, 2002; Grannas et al., 2007b) for a discussion of the state of knowledge on organics in snow packs.)

Snow and ice are important substrates for a range of atmospherically relevant reactions (Domine and Shepson, 2002; Grannas et al., 2007b). A number of laboratory studies have investigated the photolysis of inorganic (primarily $\mathrm{OH}-$ producing) compounds (Dubowski et al., 2001, 2002; Qiu et al., 2002; Chu and Anastasio 2003; Cotter et al., 2003; Chu and Anastasio 2005; Jacobi et al., 2006; Anastasio et al., 2007; Chu and Anastasio, 2007; Anastasio and Chu, 2009), as well as photolabile organic compounds (Dubowski and Hoffmann, 2000; Klan and Holoubek, 2002; Klan et al., 2003; Klanova et al., 2003a, b; Grannas et al., 2007a; Kahan and Donaldson 2007; Ram and Anastasio, 2009; Kahan et al., 2010) in snow and ice under conditions relevant to Earth's surface. A handful of experiments (Klanova et al., 2003a; Grannas et al., 2007a; Kahan and Donaldson, 2007, 2008; Matykiewiczova et al., 2007; Ram and Anastasio 2009) have examined bimolecular reactions in or at the surface of snow and ice. Some studies (Chu and Anastasio, 2003, 2005, 2007; Cotter et al., 2003; Jacobi et al., 2006; Anastasio and Chu, 2009; Ram and Anastasio, 2009) indicate that reaction kinetics are similar in ice and in aqueous solution, while others (Dubowski and Hoffmann, 2000; Klanova

Published by Copernicus Publications on behalf of the European Geosciences Union. 
et al., 2003a; Grannas et al., 2007a; Kahan and Donaldson, 2007, 2008; Kahan et al., 2010) suggest that reactivity is different in the two media. Although treating ice as a similar reaction medium to liquid water makes modelling the chemical fate of species in ice straightforward, laboratory results do not necessarily support this approach. While similar reaction rates for $\mathrm{OH}$ with the PAHs phenanthrene, pyrene, and fluoranthene have been reported in water and ice (Ram and Anastasio, 2009), degradation of monochlorophenols in the presence of hydroxyl radicals is reported to occur more slowly in ice compared to in aqueous solution (Klanova et al., 2003a), and anthracene was not observed to react with $\mathrm{OH}$ at air-ice interfaces (Kahan and Donaldson 2007).

Some of the contradictions in the above-mentioned laboratory results could be due to the heterogeneity of ice in laboratory-prepared samples and in the natural environment. Specifically, it may be that the reactivity of PAHs is different in the two parts of ice where reactions are most likely to occur: Liquid regions such as veins and pockets within bulk ice, and in the disordered region at the air-ice interface referred to as the quasi-liquid layer (QLL). (For a discussion of the physical properties of ice as they relate to snowpack photochemistry, see (Domine et al., 2008)).

The idea that the bulk and the surface of ice present two distinct reaction environments is based on the observation that the photolysis rates of anthracene and naphthalene (Kahan and Donaldson, 2007) measured in situ at air-ice interfaces were greatly enhanced compared to the rates in solution, whereas the photolysis rates of phenanthrene, pyrene, and fluoranthene (Ram and Anastasio 2009) measured by analyzing melted ice samples were not. To test this hypothesis, we measured (Kahan et al., 2010) photolysis kinetics of anthracene solutions frozen into large ice cubes, as well as the kinetics when the ice cubes were crushed to yield higher surface area-to-volume ratios under identical illumination conditions. Photolysis proceeded at a similar rate in the ice cubes as in room temperature aqueous solution, but the rate in the crushed ice was approximately four times faster. This enhanced rate matched that previously measured directly at airice interfaces using the same illumination system (Kahan and Donaldson, 2007).

The results described above confirm that photoreactivity in the QLL can be different from that in liquid regions present within bulk ice. In this work we test a hypothesis that the reactivity of $\mathrm{OH}$ toward organic compounds is also different in these two locations. If the production rate or reactivity of $\mathrm{OH}$ is suppressed at air-ice interfaces compared to in bulk ice, the discrepancy in indirect photolysis rates of PAHs measured in the QLL (Kahan and Donaldson, 2007) and in bulk ice (Ram and Anastasio 2009) could be resolved. To explore this possibility, here we irradiated aqueous benzene solutions containing the photochemical $\mathrm{OH}$ precursors $\mathrm{NO}_{2}^{-}$, $\mathrm{NO}_{3}^{-}$, and $\mathrm{H}_{2} \mathrm{O}_{2}$, and compared phenol formation kinetics in aqueous solution, ice cubes, and crushed ice to determine the effect of the reagents' location in ice on the observed reaction kinetics. We further performed real-time measurements of the same reactions in situ in solution and in the QLL, and also monitored the heterogeneous reactions between $\mathrm{OH}(\mathrm{g})$ and benzene or anthracene at air-ice interfaces. Together, the results show that while the reactivity of hydroxyl radicals toward aromatic species is similar in bulk ice to that measured in, or at the surface of, aqueous solution, it is significantly suppressed at air-ice interfaces.

\section{Methods}

\subsection{Overview}

In this study, we explored the formation of $\mathrm{OH}$. While benzene has been used as a trap to measure $\mathrm{OH}$ formation rates in natural waters (e.g. Vione et al., 2006), and in acetonitrilewater mixtures at temperatures below $0{ }^{\circ} \mathrm{C}$ (Galbavy et al., 2007), it has not previously been used as an in situ, real time probe. It has also, to our knowledge, never been used in frozen samples.

Several types of complementary experiments were performed:

(a) Offline measurements of phenol formed from the reaction of benzene with photoformed $\mathrm{OH}$ : Aqueous samples containing benzene and $\mathrm{H}_{2} \mathrm{O}_{2}$ were frozen. Ice samples with varying surface area-to-volume ratios were irradiated and the phenol formation rates were contrasted with those in aqueous solution under the same conditions.

(b) Development of an in situ probe to measure $\mathrm{OH}$ reactivity via reaction with benzene: We developed and validated a technique to measure phenol formation rates in situ in aqueous solution and at air-ice interfaces using laser-induced fluorescence (LIF).

(c) Use of this probe to monitor $\mathrm{OH}$ in aqueous solution: We used the in situ technique we developed to measure phenol formation rates in aqueous solution due to the photolysis of $\mathrm{H}_{2} \mathrm{O}_{2}, \mathrm{NO}_{3}^{-}$, and $\mathrm{NO}_{2}^{-}$. We used our results to calculate the photon flux of our lamp, which was compared to the predicted flux to further validate the in situ technique.

(d) Extension of the measurements to air-ice interfaces: We monitored phenol formation at air-ice interfaces during photolysis of hydroxyl radical precursors.

(e) In situ measurements of reactions between gas-phase $\mathrm{OH}$ and aromatics at liquid or solid aqueous interfaces: We formed $\mathrm{OH}$ in the gas phase through $\mathrm{HONO}$ photolysis, and monitored its reaction with benzene or anthracene present at air-water and air-ice interfaces. 
Table 1. Excitation and emission wavelengths for spectra in solution and at the air-ice interface.

\begin{tabular}{llcc}
\hline Sample Description & Spectrum Type & Excitation Wavelength(s) (nm) & Emission Wavelength(s) (nm) \\
\hline Benzene (solution) & Excitation $^{\mathrm{a}}$ & $245-275$ & 318 \\
& Emission $^{\mathrm{a}}$ & 261 & $264-472$ \\
Benzene (ice) & Excitation $^{\mathrm{b}}$ & $245-315$ & 318 \\
& Emission $^{\mathrm{b}}$ & 261 & $284-472$ \\
Phenol (solution) & Excitation $^{\mathrm{a}}$ & $245-289$ & 318 \\
& Emission $^{\mathrm{a}}$ & 271 & $264-472$ \\
Phenol (ice) & Excitation $^{\mathrm{b}}$ & $245-315$ & 318 \\
& Emission $^{\mathrm{b}}$ & 261 & $284-472$ \\
\hline
\end{tabular}

a Spectra were acquired using both LIF (or glancing-angle LIF) and with the commercial fluorimeter;

${ }^{b}$ Spectra were acquired only with LIF (or glancing-angle LIF)

\subsection{Offline measurements: experiment set (a)}

\subsubsection{Sample preparation}

Aqueous solutions containing $1.1 \times 10^{-3} \mathrm{~mol} \mathrm{~L}^{-1}$ benzene and $9.9 \times 10^{-4} \mathrm{~mol} \mathrm{~L}^{-1} \quad \mathrm{H}_{2} \mathrm{O}_{2}$ were prepared daily. Ice cubes were prepared (Kahan et al., 2010) by freezing $3 \mathrm{~mL}$ aliquots of aqueous solutions into semi-spheres with radii of $\sim 1.13 \mathrm{~cm}$ in an ice cube tray. Ice granules with radii of $\sim 1.5 \mathrm{~mm}$ were prepared by crushing the ice cubes. The samples were crushed on the chilled floor of the reaction chamber described below to prevent melting. Given the sample volumes and radii, we estimate that the surface area-to-volume ratio of the ice granules was approximately five times greater than that of the ice cubes. The $\mathrm{pH}$ of the samples was not adjusted, and so was likely $\sim \mathrm{pH} 5.5$ in all cases.

\subsubsection{Experimental details}

Samples were irradiated by the output of a $75 \mathrm{~W}$ xenon arc lamp which passed through a $295 \mathrm{~nm}$ longpass cutoff filter. Aqueous samples were irradiated at room temperature in $10 \mathrm{~mm}$ pathlength quartz cuvettes. The lamp was situated approximately $50 \mathrm{~cm}$ from the sample, and the beam was centered on the cuvette. Solutions were irradiated for up to two hours. At known time intervals, the samples were removed from the light and excitation spectra were acquired using a commercial fluorimeter. Excitation and emission wavelengths for all spectra are detailed in Table 1.

Ice cubes and ice granules were placed on a stainless steel plate in the center of an aluminum reaction chamber with a copper floor which was maintained at approximately $-16^{\circ} \mathrm{C}$ by copper cooling tubes. The temperature inside the chamber was maintained at approximately $-16^{\circ} \mathrm{C}$. The lamp's output was directed by a first-surface mirror with maximum reflectance at $355 \mathrm{~nm}$ into the chamber through an opening in its roof so that the sample was evenly illuminated. The distance between the lamp and the sample was approximately $75 \mathrm{~cm}$. Samples were irradiated for a known time period, then melted into brown glass bottles and stored in the dark until analysis. Excitation spectra were acquired in quartz cuvettes. Analysis was always performed on the same day as photolysis.

Reaction kinetics between benzene and $\mathrm{OH}$ were obtained by measuring the growth rate of phenol during photolysis. At longer irradiation times, phenol formed in the initial reaction can go on to react with $\mathrm{OH}$. To avoid this complication, we determined phenol's initial growth rate by the slope of the linear fit to the concentration vs. time data at short irradiation times. Figure 1 shows excitation and emission spectra of benzene and phenol in aqueous solution. The emission spectra of the two species overlap almost entirely, but phenol absorbs at wavelengths above $270 \mathrm{~nm}$, whereas benzene does not. To minimize interferences from benzene, we monitored the growth of phenol intensity using $271 \mathrm{~nm}$ as the excitation wavelength and $318 \mathrm{~nm}$ for emission, as indicated by the arrows in the figure. Excitation spectra were acquired at a scan rate of $100 \mathrm{~nm} \mathrm{~min}{ }^{-1}$ and with slit widths of $4.5 \mathrm{~nm}$. Emission intensity was related to phenol concentration using a Beer-Lambert plot, as described in the supplementary information http://www.atmos-chem-phys.net/10/843/2010/ acp-10-843-2010-supplement.pdf.

\subsection{In situ measurements: experiment sets (b)-(e)}

\subsubsection{Sample preparation}

For the experiments in (b), aqueous solutions containing $10^{-6}$ to $10^{-3} \mathrm{~mol} \mathrm{~L}^{-1}$ benzene were prepared daily. Ice samples for the in situ QLL measurements were prepared as described previously (Kahan and Donaldson 2007). Approximately $1 \mathrm{~mL}$ of aqueous solution was frozen on a stainless steel plate in a Teflon chamber with a copper floor under which copper cooling tubes run. After freezing, the ice was inverted to present a flat surface to the laser. The temperature of the chamber floor was maintained at approximately $-16^{\circ} \mathrm{C}$. To ensure that benzene was present at the air-ice interface, some ice samples were prepared by freezing pure 

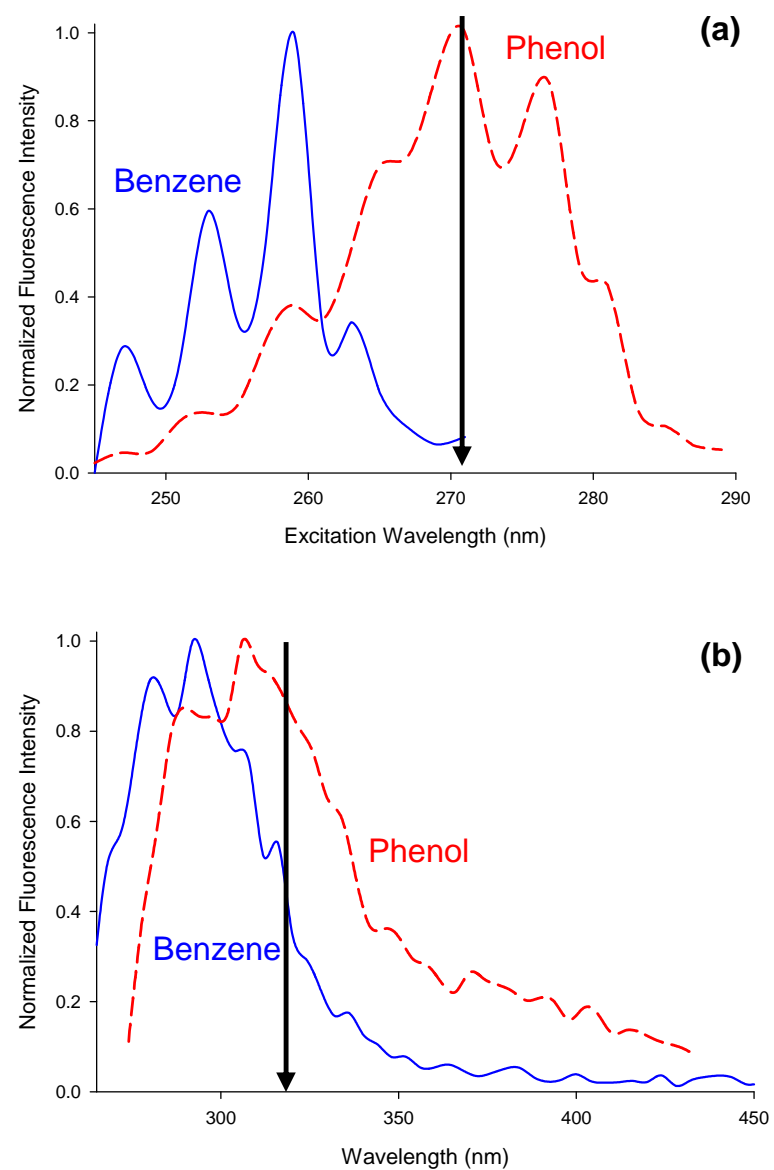

Fig. 1. (a) Excitation, and (b) emission spectra of aqueous solutions of benzene $\left(2 \times 10^{-3} \mathrm{~mol} \mathrm{~L}^{-1}\right)$ and phenol $\left(4 \times 10^{-4} \mathrm{~mol} \mathrm{~L}^{-1}\right)$ at room temperature measured with LIF. The arrows in (a) and (b) respectively show the excitation and emission wavelengths used to monitor kinetics.

water and then depositing benzene to the ice surface from the gas phase by sampling the headspace above liquid benzene using a Pasteur pipette, and "puffing" it onto the ice surface. For some experiments, molecular oxygen was removed from the ice sample by flushing the chamber with nitrogen during sample freezing and irradiation; the nitrogen entered and exited the chamber through ports in the walls.

For (c), aqueous solutions containing $1.2 \times 10^{-3} \mathrm{~mol} \mathrm{~L}^{-1}$ benzene and $10^{-5}$ to $10^{-3} \mathrm{~mol} \mathrm{~L}^{-1} \mathrm{NaNO}_{2}, \mathrm{H}_{2} \mathrm{O}_{2}$, or $\mathrm{HNO}_{3}$ as $\mathrm{OH}$ precursors were prepared daily. Ice samples for (d) were prepared as in (b), with benzene concentrations ranging from $10^{-6}$ to $10^{-3} \mathrm{~mol} \mathrm{~L}^{-1}$, and $\mathrm{OH}$-precursor concentrations ranging from $10^{-5}$ to $10^{-3} \mathrm{~mol} \mathrm{~L}^{-1}$. The reagent concentrations were varied significantly to determine whether there was a concentration dependence to the results. For some experiments, both benzene and nitric acid were introduced to the ice surface from the gas phase. Nitric acid was introduced in a similar fashion as benzene, by sampling the headspace above a concentrated $\mathrm{HNO}_{3}$ solution (Kahan and Donaldson 2007).
Aqueous solutions for (e) contained either $1.2 \times 10^{-3} \mathrm{~mol} \mathrm{~L}^{-1}$ benzene or $9.0 \times 10^{-8} \mathrm{~mol} \mathrm{~L}^{-1}$ anthracene. Ice samples containing benzene were prepared as in (b). Anthracene was deposited onto pure ice surfaces from the gas phase (Kahan and Donaldson 2007) by flowing dry $\mathrm{N}_{2}$ at a flow rate of $1 \mathrm{~L} \mathrm{~min}^{-1}$ through a two-necked round bottom flask containing several grams of anthracene, which was immersed in a water bath heated to $\sim 50^{\circ} \mathrm{C}$. The saturated nitrogen stream passed through $\sim 50 \mathrm{~cm}$ of PTFE tubing before entering the chamber through a port in the wall. Deposition of anthracene continued for approximately $15 \mathrm{~min}$; anthracene's fluorescence intensity was monitored during deposition to ensure that its surface coverage remained in the non-saturated (i.e. submonolayer) regime (Mmereki and Donaldson, 2003; Kahan and Donaldson, 2007).

Hydroxyl radicals were produced from the photolysis of gas-phase HONO. Nitrogen gas was bubbled through a concentrated $\mathrm{HCl}$ solution, then passed over glass beads coated with damp $\mathrm{NaNO}_{2}$ (Febo et al., 1995; Aubin and Abbatt, 2007). The HONO thus formed was immediately introduced to the chamber through a port in the wall. The $\mathrm{pH}$ of the samples was not adjusted for any of the experiments, and so ranged from being quite acidic (for samples containing nitric acid) to around $\mathrm{pH}$ 5.5.

\subsubsection{Experimental details}

In situ analysis was performed using LIF. The laser used for excitation was either a Nd:YAG-pumped optical parametric oscillator (OPO), which was tunable in the UV between 245 and $355 \mathrm{~nm}$, or a nitrogen laser with emission at $337 \mathrm{~nm}$. Both lasers were operated at $10 \mathrm{~Hz}$. The OPO output pulses were $\sim 4 \mathrm{~ns}$ in duration with pulse energies of $\sim 1.5 \mathrm{~mJ}$ and the $\mathrm{N}_{2}$ laser pulses were $3 \mathrm{~ns}$ long, with energies of $300 \mu \mathrm{J}$. Emission was collected through a liquid light guide (LLG) oriented perpendicular to the laser beam and passed through a monochromator, then was detected by a photomultiplier tube and sent to a digital oscilloscope for analysis. A $100 \mathrm{~ns}$ slice of the fluorescence decay was captured and averaged at each wavelength.

The supplementary information http: //www.atmos-chem-phys.net/10/843/2010/ acp-10-843-2010-supplement.pdf displays schematics which show complete details of the experimental conditions in each experiment. For aqueous samples in (b) and (c), the laser beam was oriented horizontally, and the LLG was positioned horizontally to collect emission from the side of the quartz cuvette. All ice samples, as well as aqueous samples in (e), were contained in the reaction chamber. The laser beam entered the chamber such that it impinged upon the sample surface at a very shallow angle $\left(\sim 3^{\circ}\right.$ from horizontal) (Kahan and Donaldson, 2007). The LLG was suspended $\sim 1 \mathrm{~cm}$ above the sample surface. This geometry affords us surface sensitivity (Kahan et al., 2007) and 
has allowed us to monitor reactions at air-ice (Kahan and Donaldson, 2007, 2008; Kahan et al., 2010) and air-water (Mmereki and Donaldson, 2003; Clifford et al., 2007) interfaces.

The surface thickness of the QLL is a subject of much debate. It is likely no greater than $100 \mathrm{~nm}$ in thickness, although it could be as shallow as a few Angstroms. In this work, we are not concerned with its thickness, but solely with the fact that it appears to present a reaction environment that is different from bulk ice or aqueous solution. We have previously (Kahan and Donaldson, 2007) measured identical anthracene photolysis kinetics on ice surfaces whether anthracene was introduced to the ice surface from the gas phase (in which case all anthracene is expected to react at the ice surface) or whether it was frozen from solution (in which case reactions are expected to occur at the ice surface and in the ice bulk). The identical kinetics suggest that we do not measure significant contributions from the ice bulk using this technique.

In the current work, for some experiments we introduced one or both reagents to ice samples from the gas phase, thus ensuring that they were present - and reacted - at the surface. Ensuring that reactions are occurring at aqueous surfaces is more difficult, as diffusion into the bulk is possible. We have previously demonstrated (Mmereki and Donaldson, 2003) that anthracene partitions extensively to aqueous surfaces, and that reactions with gas-phase compounds occur at the surface, as opposed to in the bulk. Therefore, we are satisfied that anthracene reacted with $\mathrm{OH}$ at the water surface. We have not performed experiments to determine the extent to which benzene partitions to aqueous surfaces. Therefore, it is possible that at least some of the phenol formation we observed during the reaction with gas-phase $\mathrm{OH}$ occurred in the bulk. However, we do not believe this affects our conclusions, as we have confidence that the reaction with anthracene does occur primarily at the air-water interface.

Phenol concentrations in aqueous solution were monitored by exciting samples at $271 \mathrm{~nm}$ and monitoring emission at $318 \mathrm{~nm}$, as in the offline experiments. The in situ spectra were identical to those measured offline using the fluorimeter. However, as shown in Fig. 2, the excitation and emission spectra of benzene and phenol at air-ice interfaces are quite different from those in aqueous solution, likely due to self-association of benzene and phenol at the air-ice interface (Kahan and Donaldson, 2007, 2008; Ardura et al., 2009). While the excitation spectra of benzene and phenol overlap on ice, phenol emits in the region between 300 and $320 \mathrm{~nm}$ and benzene does not. Therefore, for the experiments in (d) and (e), we monitored phenol formation at air-ice interfaces by exciting our ice samples at $261 \mathrm{~nm}$ and detecting emission at $318 \mathrm{~nm}$, as indicated by the arrows in the figure. In (e), anthracene loss kinetics at air-water and air-ice interfaces were measured by exciting the samples at $337 \mathrm{~nm}$ and monitoring emission at $405 \mathrm{~nm}$ (Mmereki and Donaldson, 2003; Kahan and Donaldson, 2007).
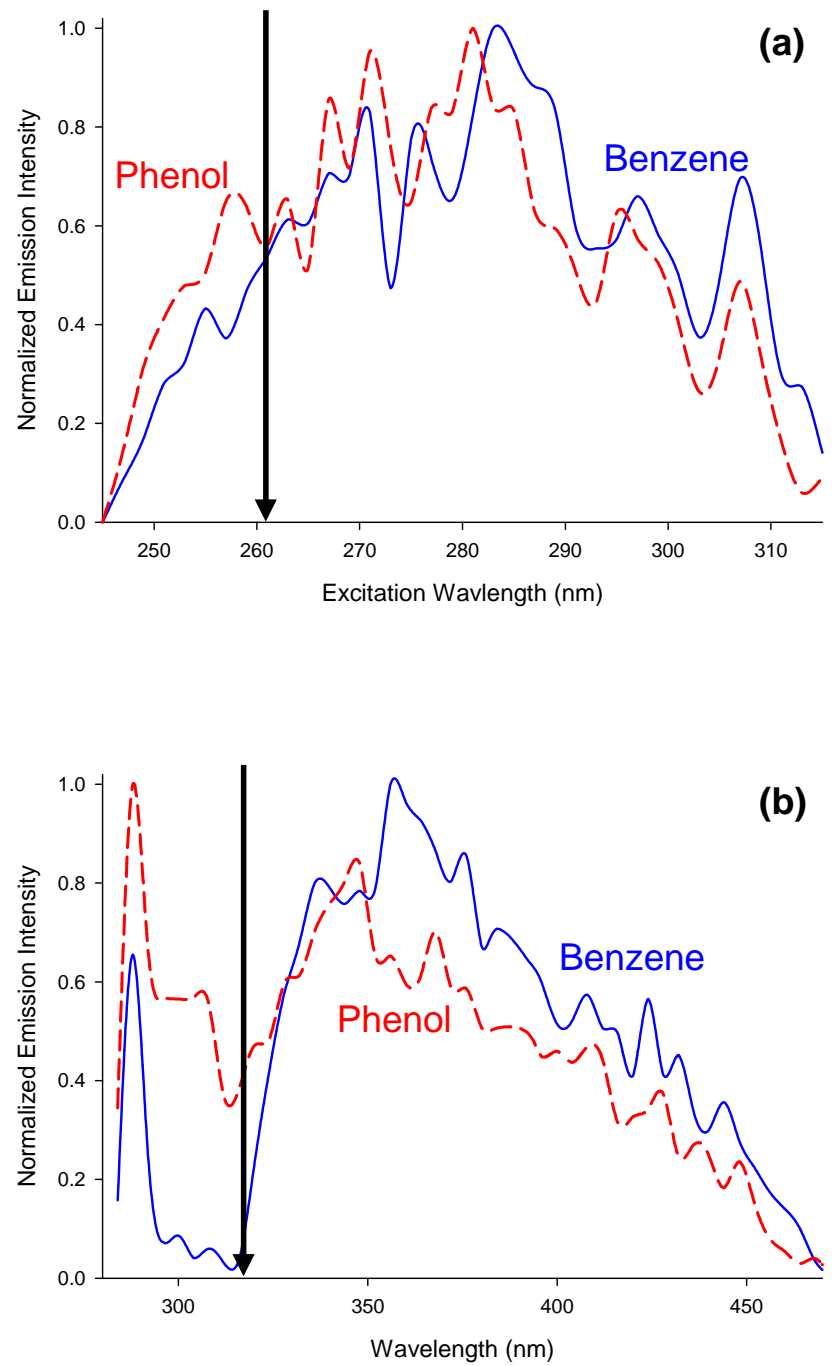

Fig. 2. (a) Excitation and (b) emission spectra of frozen aqueous solutions of benzene $\left(2 \times 10^{-3} \mathrm{~mol} \mathrm{~L}^{-1}\right)$ and phenol $\left(4 \times 10^{-5} \mathrm{~mol} \mathrm{~L}^{-1}\right)$ acquired at air-ice interfaces using glancingangle LIF. The sharp peaks in the emission spectra at $\sim 285 \mathrm{~nm}$ are due to Raman scattering from the ice. The arrows in (a) and (b) respectively show the excitation and emission wavelengths used to monitor kinetics.

In (b), aqueous benzene solutions were contained in $10 \mathrm{~mm}$ pathlength quartz cuvettes and were irradiated at room temperature with either the $261 \mathrm{~nm}$ output of the OPO, the full output of a $100 \mathrm{~W}$ xenon arc lamp, or both. The lamp's output was directed by a first-surface mirror with maximum reflectance at $355 \mathrm{~nm}$ onto the sample at a downward angle such that the entire sample was irradiated uniformly. At known time intervals, the laser's output was tuned to $271 \mathrm{~nm}$ and phenol's emission intensity was recorded. Ice samples were contained in the reaction chamber, and were irradiated by the $261 \mathrm{~nm}$ output of the OPO, by the full output of the $100 \mathrm{~W}$ lamp, or by both. The lamp's output was 
directed by a first-surface mirror onto the sample through a quartz window in the chamber roof. Phenol emission intensity was recorded automatically every $20 \mathrm{~s}$. The distance between the lamp and the samples was $\sim 90 \mathrm{~cm}$ for all in situ experiments.

In (c) and (d), samples were irradiated with the output of the $100 \mathrm{~W}$ xenon arc lamp which passed through a $295 \mathrm{~nm}$ longpass cutoff filter. The lamp geometries were identical to those in (b). For (c), LIF intensity was collected automatically every $20 \mathrm{~s}$. For (d), the laser was blocked during photolysis by the lamp, and the lamp was blocked during data acquisition.

In (e), both aqueous and ice samples were contained in the reaction chamber. Aqueous samples were contained either in a Petri dish or a stainless steel plate with $2 \mathrm{~mm}$ high "walls" made of silicone vacuum grease, and ice samples were placed on a stainless steel plate which covered the chamber floor to prevent oxidation of the copper. Gas-phase HONO was introduced to the chamber and then photolyzed to form $\mathrm{OH}$. This was done either by directing the wavelength-filtered output of the $100 \mathrm{~W}$ xenon arc lamp onto the sample surface through the quartz window in the chamber roof, or by introducing the unfiltered output of the lamp into the chamber horizontally through a quartz window in the back of the chamber such that the sample itself was not directly illuminated. LIF measurements of phenol for aqueous and ice samples were performed as in (c) and (d), and LIF measurements of anthracene were performed by exciting the sample with the $\mathrm{N}_{2}$ laser and measuring emission intensity at $405 \mathrm{~nm}$ automatically every $20 \mathrm{~s}$.

\subsection{Reagents}

All reagents were used as purchased without further purification. Benzene (purity $\geq 99.0 \%$ ) and $\mathrm{H}_{2} \mathrm{O}_{2}$ (lab grade) were purchased from ACP. Anthracene (99\% purity) and $\mathrm{NaNO}_{2}$ (Reagent Plus) were from Sigma Aldrich. Nitric acid was from Caledon, and $\mathrm{HCl}$ was from Baker. Nitrogen gas (99.998\% purity) was from B.O.C. $18 \mathrm{M} \Omega$ deionized water was used for all experiments.

\section{Results}

\subsection{Experiment set (a): offline measurements}

The aim of this set of experiments was to determine whether the production or reactivity of $\mathrm{OH}$ is different at air-ice interfaces than it is in liquid regions within bulk ice and in aqueous solution. We used benzene as an $\mathrm{OH}$ trap and measured phenol production rates following the photolysis of $\mathrm{H}_{2} \mathrm{O}_{2}$ as an $\mathrm{OH}$ source in aqueous solution, ice cubes, and ice granules. Full details of the $\mathrm{OH}$-trap are given in the supplementary information http://www.atmos-chem-phys. net/10/843/2010/acp-10-843-2010-supplement.pdf. Figure 3 shows phenol concentration as a function of irra-

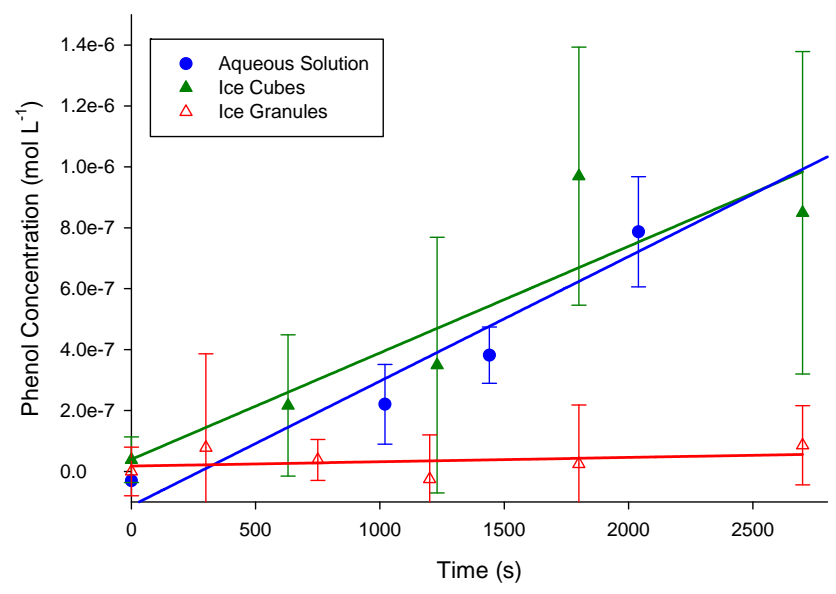

Fig. 3. Phenol concentration as a function of irradiation time for samples containing $1 \times 10^{-3} \mathrm{~mol} \mathrm{~L}^{-1}$ benzene and $9 \times 10^{-4} \mathrm{~mol} \mathrm{~L}^{-1} \mathrm{H}_{2} \mathrm{O}_{2}$, measured offline by fluorimetry. The solid traces are linear fits to the data, and error bars represent one standard deviation about the mean for at least three trials.

diation time in the three media. The phenol production rates in aqueous solution and in ice cubes were the same within error $\left((4.2 \pm 0.9) \times 10^{-10} \mathrm{~mol} \mathrm{~L}^{-1} \mathrm{~s}^{-1}\right.$ and $(4 \pm 2) \times 10^{-10} \mathrm{~mol} \mathrm{~L}^{-1} \mathrm{~s}^{-1}$ respectively). With ice granules, however, no phenol growth was observed. Based on the uncertainty in the data, we can ascribe an upper limit to the phenol formation rate in the ice granules of $9 \times 10^{-11} \mathrm{~mol} \mathrm{~L}^{-1} \mathrm{~s}^{-1}$.

These results support our suggestion (Kahan et al., 2010) that kinetics measured in the ice cubes are more representative of reactivity in liquid regions within bulk ice, and kinetics measured in the ice granules are more representative of reactivity in the QLL. The lack of phenol growth in ice granules supports our hypothesis that $\mathrm{OH}$ is either produced inefficiently at the air-ice interface, or that it reacts inefficiently with any benzene that is present there.

\subsection{Experiment set (b): validation of an in situ OH- probe}

To explore whether the results of (a) were due to different behaviour of $\mathrm{OH}$ at air-ice interfaces compared to in bulk ice and liquid water, we developed an in situ technique to measure phenol formation directly at air-ice interfaces. To test this probe's ability to measure phenol growth in real time, we irradiated aqueous and ice samples containing benzene at wavelengths below $295 \mathrm{~nm}$. Under irradiation at low wavelengths, benzene has been reported to react directly with molecular oxygen to form phenol in the gas phase (Tubaro et al., 2003) and in liquid benzene samples (Stella et al., 2008). The proposed mechanism involves ionization to form the benzene radical cation which reacts with molecular oxygen to form a peroxy cation radical intermediate; phenol is 

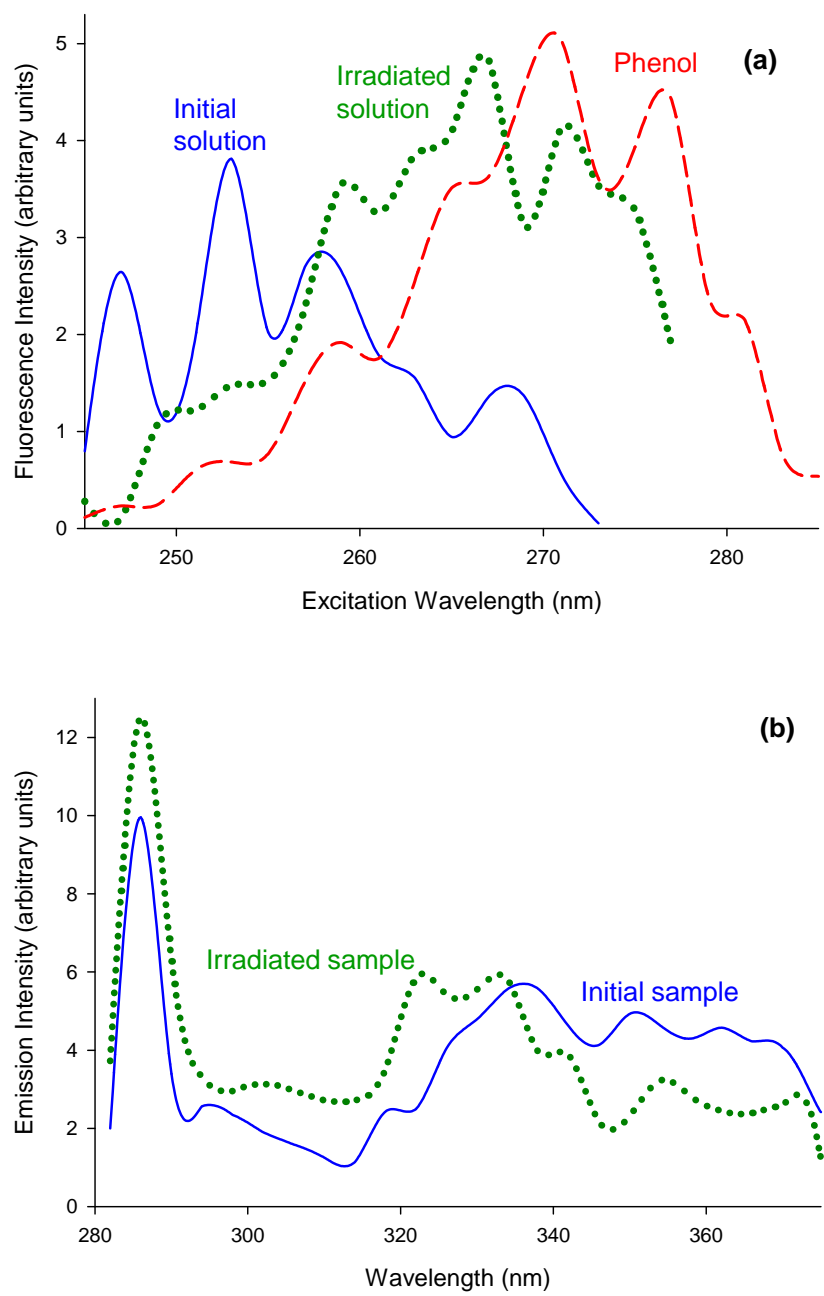

Fig. 4. (a) Excitation spectra of a room temperature aqueous solution containing $10^{-3} \mathrm{~mol} \mathrm{~L}^{-1}$ benzene prior to and after irradiation by the $261 \mathrm{~nm}$ output of the OPO, as well as an excitation spectrum of phenol in aqueous solution; (b) Glancing-angle LIF emission spectra of a frozen solution containing $10^{-5} \mathrm{~mol} \mathrm{~L}^{-1}$ benzene prior to and after irradiation by the $261 \mathrm{~nm}$ output of the OPO. The sharp peaks around $\sim 285 \mathrm{~nm}$ are due to Raman scattering from the ice.

formed by the loss of an oxygen atom (Tubaro et al., 2003). Figure 4a shows excitation spectra of an aqueous benzene sample before and after irradiation, as well as an excitation spectrum of phenol. Figure $4 \mathrm{~b}$ shows emission spectra at an ice surface for a frozen sample containing benzene before and after irradiation. The growth of phenol after irradiation is evident both on ice and in aqueous solution. The presence of phenol in the ice surface experiments was confirmed by acquiring excitation spectra of the melted samples and by HPLC analysis (see the supplementary information for details http://www.atmos-chem-phys.net/10/843/ 2010/acp-10-843-2010-supplement.pdf). The involvement of $\mathrm{O}_{2}(\mathrm{~g})$ in phenol formation was tested by flushing the solu-

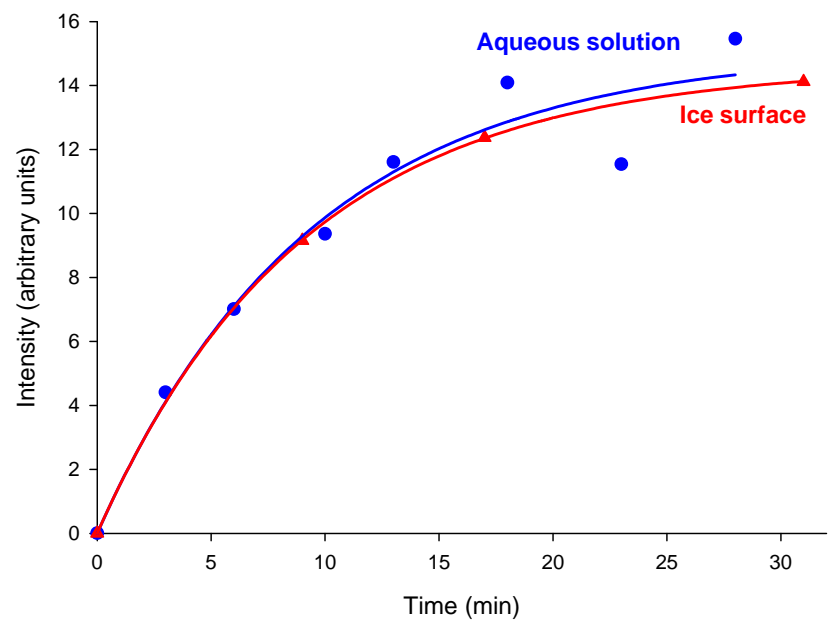

Fig. 5. Growth of phenol fluorescence intensity during irradiation by the full output of the $100 \mathrm{~W}$ xenon arc lamp of a $10^{-3} \mathrm{~mol} \mathrm{~L}^{-1}$ benzene in aqueous solution and a frozen $10^{-5} \mathrm{~mol} \mathrm{~L}^{-1}$ benzene solution at the air-ice interface measured at $318 \mathrm{~nm}$. Kinetics were measured with LIF and glancing-angle LIF. The solid traces are exponential fits to the data.

tions prior to freezing with $\mathrm{N}_{2}$, as well as flowing $\mathrm{N}_{2}$ through the reaction chamber during freezing and irradiation. No phenol growth was observed in these experiments, confirming the role of molecular oxygen in the low wavelength benzene photolysis mechanism.

Figure 5 shows the growth in the LIF signal due to phenol formation during irradiation of benzene samples in aqueous solution and at air-ice interfaces in the presence of atmospheric $\mathrm{O}_{2}$. The first-order growth rate is the same in both media. These results show that we are able to monitor the conversion of benzene to phenol in real time both in aqueous solution and at air-ice interfaces.

\subsection{Experiment set (c): photolysis of $\mathrm{OH}$-precursors in solution}

The results of (b) show that the in situ probe is capable of detecting phenol growth in aqueous solution and at air-ice interfaces in real time. In (c), we tested the ability of this technique to measure $\mathrm{OH}$ formation rates in situ in aqueous solution. We formed $\mathrm{OH}$ by irradiating aqueous solutions containing excess benzene and varying concentrations of $\mathrm{H}_{2} \mathrm{O}_{2}, \mathrm{HNO}_{3}$, or $\mathrm{NaNO}_{2}$ at wavelengths above $295 \mathrm{~nm}$. The photolysis quantum yields and absorption cross sections of these oxidants have been studied both in aqueous solution and in ice (see Chu and Anastasio, 2003, 2005, 2007 and references therein).

Figure 6 shows the growth rate of phenol as a function of $\mathrm{OH}$-precursor concentration. At the concentrations used in this work, the dependences are linear, indicating simple second order kinetics, as expected from the rate 


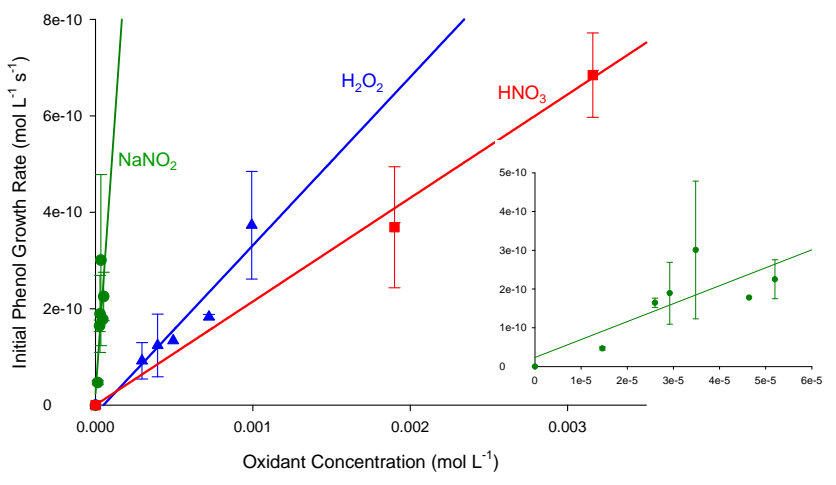

Fig. 6. Initial phenol formation rates as a function of concentration of $\mathrm{NaNO}_{2}, \mathrm{H}_{2} \mathrm{O}_{2}$, and $\mathrm{HNO}_{3}$ for photolysis in aqueous solutions containing $1 \times 10^{-3} \mathrm{~mol} \mathrm{~L}^{-1}$ benzene, measured using LIF. The solid traces are linear fits to the data. Error bars represent one standard deviation about the mean of at least two measurements. An expanded view of phenol's formation rate due to nitrite photolysis is shown in the inset.

expression $\frac{d[\mathrm{Ph}]}{d t}=k_{r x n}[\mathrm{OH}][\mathrm{B}]$. Because $\mathrm{OH}$ is formed directly from the precursor, we observe linear dependences on the precursor concentration. Using the slope of the linear fits to our data and published values of the absorption cross sections and photolysis quantum yields (Chu and Anastasio, 2003, 2005, 2007), we estimated an average photon flux for our lamp over the wavelengths of absorption of each oxidant (see the supplementary information for details http://www.atmos-chem-phys.net/10/843/ 2010/acp-10-843-2010-supplement.pdf). In each case, the calculated photon flux matched the spectral irradiance of the lamp provided by the manufacturer within a factor of three. The total irradiance of the lamp reaching our sample between 300 and $400 \mathrm{~nm}$ is approximately $4 \times 10^{13}$ photon $\mathrm{cm}^{-2} \mathrm{~s}^{-1}$, as determined from nitrite photolysis. The good agreement between the predicted spectral irradiance of our lamp and our calculated irradiance based on our measured $\mathrm{OH}$ production rates indicates that our in situ probe adequately measures $\mathrm{OH}$ production rates.

\subsection{Experiment set (d): photolysis of $\mathrm{OH}$-precursors at air-ice interfaces}

The results of (b) and (c) validated the use of our in situ technique. In (d) we monitored phenol growth at air-ice interfaces during the irradiation of frozen samples containing benzene and an $\mathrm{OH}$-precursor. Reagent concentrations were varied significantly to ensure that the lack of reactivity observed was not due to concentration effects, and in some experiments reagents were introduced from the gas phase to ensure that they were present at the ice surface. Neither reagent concentrations nor sample preparation methods had any effect on the results; under no experimental conditions did we observe a growth of intensity at $318 \mathrm{~nm}$. This result is in agreement with our previous result (Kahan and Donaldson, 2007) that indirect photolysis of anthracene, via $\mathrm{OH}$ produced from the photolysis of $\mathrm{NO}_{3}^{-}$or $\mathrm{H}_{2} \mathrm{O}_{2}$, does not occur at air-ice interfaces. This result also offers strong confirmation that the lack of phenol growth observed in the irradiated ice granules (presented in (a), above) was due to differences in reactivity at the air-ice interface compared to in aqueous solution.

To further test our hypothesis that the kinetics measured in ice granules contain contributions from reactions occurring both in bulk ice and at air-ice interfaces (with different kinetics), we estimated the relative phenol formation rates expected in our ice granules and ice cubes, using the rate constant measured in aqueous solution $\left(4.2 \times 10^{-10} \mathrm{~mol} \mathrm{~L}^{-1} \mathrm{~s}^{-1}\right)$ to describe the rate in liquid regions within bulk ice, and a rate constant at air-ice interfaces of zero. Assuming spherical granules, the rate constant associated with the surface reaction should be inversely related to the relative increase in surface area compared to that of the ice cubes. For our samples, that means that the rate in the ice granules should be approximately five times lower than in the ice cubes. This would result in a phenol formation rate of $8.4 \times 10^{-11} \mathrm{~mol} \mathrm{~L}^{-1} \mathrm{~s}^{-1}$, which is beneath our detection limits.

\subsection{Experiment set (e): heterogeneous reactions at air- water and air-ice interfaces}

The results from the previous experiments show that the photolysis of hydroxyl radical precursors in the presence of benzene does not form phenol to an appreciable extent at air-ice interfaces. This could be due to one of two reasons: The photochemical production of $\mathrm{OH}$ could be suppressed at air-ice interfaces compared to in bulk ice and aqueous solution; or hydroxyl radicals could be formed, but not react with benzene. To test the reactivity of hydroxyl radicals toward benzene at air-ice interfaces, we formed $\mathrm{OH}$ in the gas phase and monitored the heterogeneous reaction between $\mathrm{OH}$ and benzene at the surfaces of water and ice. Figure 7 shows the LIF signal intensity associated with phenol as a function of exposure time to $\mathrm{HONO}(\mathrm{g})$. During the initial stage of the experiment, when the HONO in the chamber was not irradiated, no growth of phenol was observed on water or on ice. When the lamp was turned on, the HONO photolyzed to form $\mathrm{OH}$, and an immediate growth of phenol was evident at the water surface. However, no growth was observed on ice. We performed this experiment in two ways: By irradiating the samples with the filtered $(\lambda>295 \mathrm{~nm})$ output of the lamp, and also by directing the unfiltered lamp's output just above and parallel to the samples. These two illumination geometries gave identical results.

We also measured the degradation rate of anthracene at air-water and air-ice interfaces due to heterogeneous reaction with $\mathrm{OH}$. To avoid loss of anthracene due to direct photolysis, we irradiated the chamber with the output of the unfiltered lamp oriented such that the sample itself was not illuminated. 


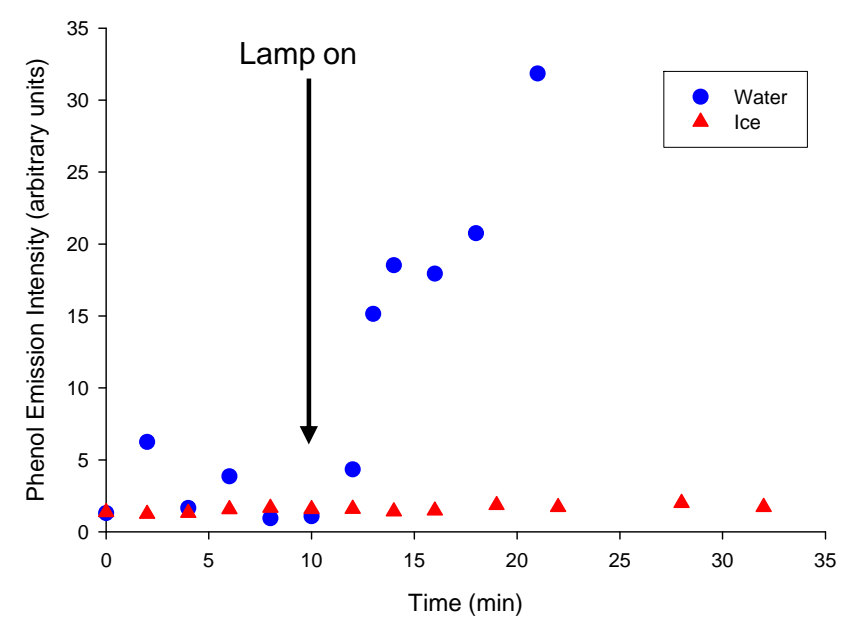

Fig. 7. Phenol fluorescence intensity during the exposure of benzene to gas-phase hydroxyl radicals at air-water and air-ice interfaces, measured using glancing-angle LIF. The chamber was flushed with gas-phase $\mathrm{HONO}$, which was irradiated to form $\mathrm{OH}$ at $t=10 \mathrm{~min}$, as indicated by the arrow.

Figure 8 shows anthracene loss as a function of time during exposure to gas-phase $\mathrm{OH}$. While rapid loss is observed at the air-water interface, no change in anthracene's concentration is observed at the air-ice interface.

These results do not rule out the possibility that hydroxyl radical formation proceeds at a different rate at air-ice interfaces than in liquid water or bulk ice. They do, however, clearly show that hydroxyl radicals are much less reactive toward benzene and anthracene at air-ice interfaces than at airwater interfaces, whether the hydroxyl radicals are formed in the QLL through the photolysis of $\mathrm{NO}_{2}^{-}, \mathrm{NO}_{3}^{-}$, or $\mathrm{H}_{2} \mathrm{O}_{2}$, or introduced from the gas phase.

\section{Discussion}

There are several possible explanations for a reduction in reactivity between $\mathrm{OH}$ and an organic compound at the airice interface. The first possibility which must be addressed is that the lack of observed reaction could be due to an experimental artefact. Here we consider two types of artefacts which could affect our measurements. The first possibility is that the glancing-angle LIF technique used may not accurately follow reactions at air-ice interfaces. We are confident that this is not the case, as we have observed the same result (a lack of reactivity of $\mathrm{OH}$ with organics at ice surfaces) using two very different techniques (in situ glancing-angle LIF measurements and offline spectroscopy of melted ice cubes and granules). Further, using the same two complementary techniques, we have measured increased anthracene photolysis rates at ice surfaces (Kahan and Donaldson, 2007; Kahan

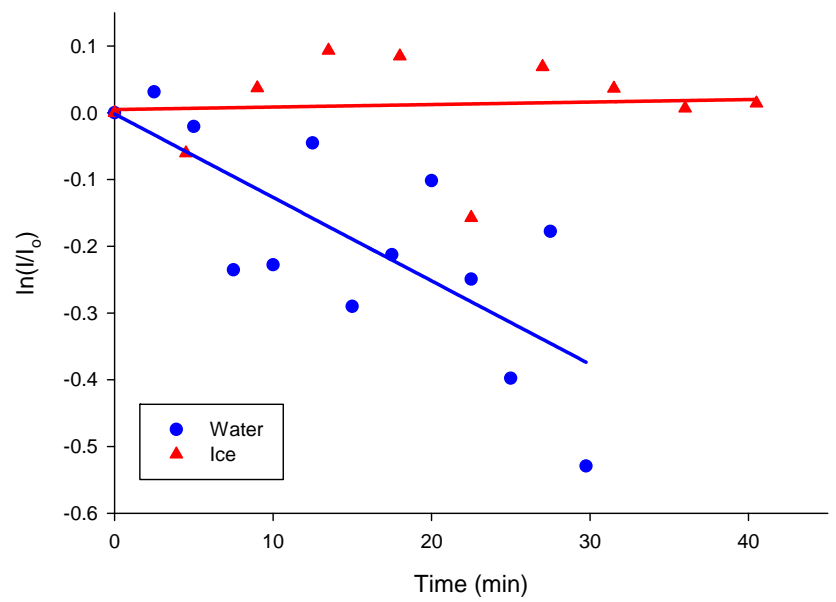

Fig. 8. Anthracene fluorescence intensity at air-water and air-ice interfaces as a function of exposure time to gas-phase $\mathrm{OH}$, measured using glancing-angle LIF. The solid traces are linear fits to the lognormalized data.

et al., 2010). We would not expect an experimental artefact to have the opposite effect on two systems.

The second artefact that we consider is that benzene could evaporate from ice surfaces and ice granules before reaction with $\mathrm{OH}$ can occur. This would explain the lack of phenol formation observed in these experiments. However, we do not believe this to be the case. Using glancing-angle LIF, we have monitored benzene at air-ice interfaces (Kahan and Donaldson in preparation), and have observed minimal loss in the dark. Under illumination conditions similar to those in this work (irradiation by a $100 \mathrm{~W}$ xenon arc lamp with a longpass $295 \mathrm{~nm}$ cutoff filter), we measure a photolysis rate constant on ice of $3 \times 10^{-4} \mathrm{~s}^{-1}$. This is much slower than the rate constants for phenol fluorescence growth measured in aqueous solution in the presence of nitrite and hydrogen peroxide, and is of similar magnitude (but generally lower) than the rate constants for phenol fluorescence growth in the presence of nitrate. These results, as well as the fact that we can observe phenol formation from the direct photolysis of benzene at air-ice interfaces (both when benzene is frozen from solution and deposited from the gas phase), indicate that the loss of benzene through processes other than phenol formation is not likely affecting our results. We are therefore confident that the lack of reactivity observed at air-ice interfaces is a real phenomenon, and is not due to an experimental artefact.

There are several possible explanations for suppressed reactivity of hydroxyl radicals at air-ice interfaces. Differences in the effective photon fluxes in the various sample types could affect measured reaction rates. However, it is unlikely that photon fluxes are reduced at air-ice interfaces and in ice granules compared to in aqueous solution or in ice cubes. If anything, we might expect enhanced photon fluxes in the ice 
granules due to increased internal reflection. Therefore, we do not believe that changes in effective photon fluxes are responsible for the lack of reaction at air-ice interfaces.

We also suggest that a temperature dependence is not responsible for the lack of reactivity on ice, as the temperature dependences of $\mathrm{OH}$ formation from $\mathrm{H}_{2} \mathrm{O}_{2}, \mathrm{NO}_{3}^{-}$, and $\mathrm{NO}_{2}^{-}$ (Chu and Anastasio, 2003, 2005, 2007) are minor. In previous work (Kahan and Donaldson, 2007), we have seen no evidence of a temperature dependence to the degradation rate of anthracene due to reaction with $\mathrm{OH}$ formed from $\mathrm{H}_{2} \mathrm{O}_{2}$ photolysis at liquid water surfaces. Although reaction pathways in ice (specifically phenol formation vs. dimerization) have been shown to be temperature dependent (Klanova et al., 2003a), this can not explain our results: We measure similar phenol formation rates in aqueous solution and in low surface-area ice cubes, which are at very different temperatures, but measure different kinetics at air-ice interfaces and in high surface-area ice granules, which are at the same temperature as the ice cubes. This strongly suggests that temperature changes are not responsible for our observations.

Since the addition of hydroxyl radicals to aromatics occurs at near diffusion-limited rates, a decrease in diffusion rates on ice could lead to slower reaction rates there. Diffusion rates are expected to decrease with decreasing temperature; however, the fact that phenol formation rates in ice cubes $\left(\right.$ at $-16^{\circ} \mathrm{C}$ ) occurs at similar rates as in aqueous solution $\left(\right.$ at $20^{\circ} \mathrm{C}$ ) indicates that any temperature dependence to the reaction (or to diffusion rates) is minimal. Further, diffusion in the QLL is expected to be quite rapid (Lee et al., 2007), so it seems unlikely that a reduction in diffusion rates is responsible for the lack of reactivity at air-ice interfaces.

It is possible that $\mathrm{OH}$ partitions poorly to ice surfaces, and is therefore not present in high enough concentrations for us to observe a reaction with benzene, whether the $\mathrm{OH}$ is formed in the ice or introduced from the gas phase. We have previously noted (Kahan and Donaldson 2008) that ozone partitions up to $75 \%$ less effectively to an air-ice interface than to a liquid water surface. However, despite its less efficient adsorption, ozone reacted much more quickly with phenanthrene on ice than on liquid water; at $50 \mathrm{ppb}$ ozone, phenanthrene's lifetime toward ozonation would be just under five days on ice, compared to 44 days on water.

Hydroxyl radicals could recombine quickly to form $\mathrm{H}_{2} \mathrm{O}_{2}$ or their parent compounds. This recombination process is thought to be a major mechanism of $\mathrm{H}_{2} \mathrm{O}_{2}$ formation in interstellar ices (Pan et al., 2004; Zheng et al., 2006). Although benzene concentrations were in excess of $\mathrm{OH}$ by at least an order of magnitude in our samples, it is still possible that $\mathrm{OH}$ self-reacted before encountering a benzene molecule. We have previously demonstrated that aromatic compounds selfassociate readily at the air-ice interface, even at submonolayer coverages (Kahan and Donaldson, 2007, 2008; Ardura et al., 2009). They likely exist in "islands" scattered across the surface, rather than as a uniform coating. It is possible that the $\mathrm{OH}$ is either formed, or adsorbed to the ice surface, in a region that is not populated by benzene. Since the recombination of $\mathrm{OH}$ in ice is thought to occur at close to diffusionlimited rates (Zheng et al., 2006), hydroxyl radicals could recombine before encountering a benzene molecule.

\section{Environmental implications}

The different reactivities observed at air-ice interfaces and in bulk ice suggest that the fate of organics in environmental snow and ice might be quite different depending on whether the compound exists primarily in liquid pockets within the ice or in the QLL. Based on the results of this study, we would expect lifetimes of organic pollutants to be much longer at air-ice interfaces compared to in bulk ice. We have previously seen, however, that direct photolysis rates of PAHs are enhanced at air-ice interfaces compared to in liquid water (Kahan and Donaldson, 2007) and bulk ice (Kahan et al., 2010). Heterogeneous ozonation of PAHs is also much faster at air-ice interfaces compared to at air-water interfaces (Kahan and Donaldson, 2008); this reaction is likely unimportant to organic compounds trapped within the ice. Given these observations, we can not draw conclusions about the lifetimes of organic pollutants in snow and ice based on their location. However, we can predict that their fate will be quite different depending on whether they are present at the air-ice interface or in bulk ice, and that in order to properly model their fate, their location must be taken into account.

As a final note, the results of this study emphasize the fact that ice is a complex, heterogeneous, and poorly understood reaction medium. Although treating it as identical to liquid water may be applicable in some cases (e.g. if the reagents are located within the ice bulk), it is clear that this is often not adequate, and may result in large errors in predicted atmospheric lifetimes. We have shown this to be true for aromatic organic compounds, but it is possible that the reactivity of inorganic species is also different at air-ice interfaces and in bulk ice. One very important atmospheric reaction is the photochemical formation of $\mathrm{OH}$ within snowpacks, which can increase $\mathrm{OH}$ mixing ratios in the overlying boundary layer by over an order of magnitude compared to what is expected based on gas-phase processes alone (Domine and Shepson, 2002). While this study and others (Chu and Anastasio, 2003, 2005, 2007) suggest that $\mathrm{OH}$ production rates from the photolysis of inorganic precursors in bulk ice are similar to those in liquid water, it is not certain that the same is true in the QLL. This is important, because snow-air exchange will be most important for $\mathrm{OH}$ formed at air-ice interfaces. If $\mathrm{OH}$ formation rates are different in bulk ice and at air-ice interfaces, this will affect our understanding not only of chemistry within snow and ice, but in the overlying atmosphere as well. 
Acknowledgements. The authors thank NSERC and CFCAS for funding. TFK thanks NSERC for a Canadian Graduate Doctoral scholarship. RZ thanks NSERC for an Undergraduate Summer Research Award. The authors thank S. A. Mabury for the use of his HPLC and D. A. Jackson for assistance with HPLC measurements.

Edited by: F. Keutsch

\section{References}

Anastasio, C. and Chu, L.: Photochemistry of Nitrous Acid (HONO) and Nitrous Acidium Ion $\left(\mathrm{H}_{2} \mathrm{ONO}^{+}\right)$in Aqueous Solution and Ice, Environ. Sci. Technol., 43(4), 1108-1114, 2009.

Anastasio, C., Galbavy, E. S., Hutterli, M. A., et al.: Photoformation of hydroxyl radical on snow grains at Summit, Greenland, Atmos. Environ., 41(24), 5110-5121, 2007.

Ardura, D., Kahan, T. F., Donaldson, D. J.: Self-association of naphthalene at the air-ice interface, J. Phys. Chem. A, 113: 7353-7359, 2009.

Aubin, D. G. and Abbatt, J. P. D.: Interaction of $\mathrm{NO}_{2}$ with hydrocarbon soot: Focus on HONO yield, surface modification, and mechanism, J. Phys. Chem. A, 111(28), 6263-6273, 2007.

Carrera, G., Fernandez, P., Vilanova, R. M., et al.: Persistent organic pollutants in snow from European high mountain areas, Atmos. Environ., 35(2), 245-254, 2001.

Chu, L. and Anastasio, C.: Quantum yields of hydroxyl radical and nitrogen dioxide from the photolysis of nitrate on ice, J. Phys. Chem., 107, 9594-9602, 2003.

Chu, L. and Anastasio, C.: Formation of hydroxyl radical from the photolysis of frozen hydrogen peroxide, J. Phys. Chem. A, 109, 6264-6271, 2005.

Chu, L. and Anastasio, C.: Temperature and wavelength dependence of nitrite photolysis in frozen and aqueous solutions, Environ. Sci. Technol., 41(10), 3626-3632, 2007.

Cincinelli, A., Stortini, A. M., Checchini, L., et al.: Enrichment of organic pollutants in the sea surface microlayer (SML) at Terra Nova Bay, Antarctica: influence of SML on superfacial snow composition, J. Environ. Monitor., 7(12), 1305-1312, 2005.

Clifford, D., Bartels-Rausch, T., Donaldson, D. J.: Suppression of aqueous surface hydrolysis by monolayers of short chain organic amphiphiles, Phys. Chem. Chem. Phys., PCCP 9, 1362-1369, 2007.

Compoint, M., Toubin, C., Picaud, S., et al.: Geometry and dynamics of formic and acetic acids adsorbed on ice, Chem. Phys. Lett., 365(1-2), 1-7, 2002.

Cotter, E. S. N., Jones, A. E., Wolff, E. W., et al.: What controls photochemical $\mathrm{NO}$ and $\mathrm{NO}_{2}$ production from Antarctic snow? Laboratory investigation assessing the wavelength and temperature dependence, J. Geophys. Res., 108, 8-1-8-10, 2003.

Daly, G. L. and Wania, F.: Organic contaminants in mountains, Environ. Sci. Technol., 39(2), 385-398, 2005.

Domine, F., Albert, M., Huthwelker, T., et al.: Snow physics as relevant to snow photochemistry, Atmos. Chem. Phys., 8(2), 171208, 2008

Domine, F. and Shepson, P. B.: Air-snow interactions and atmospheric chemistry, Science, 297 1506-1510, 2002.

Dubowski, Y., Colussi, A. J., Boxe, C., et al.: Monotonic increase of nitrite yields in the photolysis of nitrate in ice and water between 238 and 294 K, J. Phys. Chem. A, 106(30), 6967-6971, 2002.
Dubowski, Y., Colussi, A. J., and Hoffmann, M. R.: Nitrogen dioxide release in the $302 \mathrm{~nm}$ band photolysis of spray-frozen aqueous nitrate solutions. Atmospheric implications, J. Phys. Chem. A, 105, 4928-4932, 2001.

Dubowski, Y. and Hoffmann, M. R.: Photochemical transformations in ice: Implications for the fate of chemical species, Geophys. Res. Lett., 27, 3321-3324, 2000.

Febo, A., Perrino, C., Gherardi, M., et al.: Evaluation of a HighPurity and High-Stability Continuous Generation System for Nitrous-Acid.” Environ. Sci. Technol., 29(9): 2390-2395, 1995.

Fernandez, P., G. Carrera, Grimalt, J. O., et al.: Factors governing the atmospheric deposition of polycyclic aromatic hydrocarbons to remote areas." Environ. Sci. Technol., 37(15), 3261-3267, 2003.

Franz, T. P. and Eisenreich, S. J.: Accumulation of polychlorinated biphenyls and polycyclic aromatic hydrocarbons in the snowpack of Minnesota and Lake Superior." Journal of Great Lakes Research 26(2), 220-234, 2000.

Galbavy, E. S., Anastasio, C., Lefer, B. L., et al.: Light penetration in the snowpack at Summit, Greenland: Part I - Nitrite and hydrogen peroxide photolysis, Atmos. Environ., 41(24), 50775090, 2007.

Grannas, A. M., Bausch, A. R., et al.: Enhanced aqueous photochemical reaction rates after freezing, J. Phys. Chem. A, 111(43), 11043-11049, 2007a.

Grannas, A. M., Jones, A. E., Dibb, J., et al.: An overview of snow photochemistry: evidence, mechanisms and impacts, Atmos. Chem. Phys., 7(16), 4329-4373, 2007b.

Herbert, B. M. J., Villa, S., et al.: Chemical interactions with snow: Understanding the behavior and fate of semi-volatile organic compounds in snow, Ecotoxicology and Environmental Safety, 63(1), 3-16, 2006.

Jacobi, H. W., Annor, T., Quansah, E., et al.: Investigation of the photochemical decomposition of nitrate, hydrogen peroxide, and formaldehyde in artificial snow, J. Photochem. Photobiol., 179(3), 330-338, 2006.

Jaffrezo, J. L., M. P. Clain, and Masclet, P.: Polycyclic AromaticHydrocarbons in the Polar Ice of Greenland - Geochemical Use of These Atmospheric Tracers, Atmos. Environ., 28(6), 1139$1145,1994$.

Kahan, T. F. and Donaldson, D. J.: Photolysis of polycyclic aromatic hydrocarbons on water and ice surfaces, J. Phys. Chem. A, 111, 1277-1285, 2007.

Kahan, T. F. and Donaldson, D. J.: Heterogeneous ozonation kinetics of phenanthrene at the air-ice interface, Environ. Res. Lett. 3, 045006z, doi:10.1088/1748-9326/3/4/045006, 2008.

Kahan, T. F., Reid, J. P., and Donaldson, D. J.: Spectroscopic probes of the quasi-liquid layer on ice, J. Phys. Chem. A, 111(43), 11006-11012, 2007.

Kahan, T. F. and Donaldson, D. J.: Benzene photolysis at air-ice interfaces: Implications for the fate of organic pollutants in the winter, in preparation, 2010.

Kahan, T. F., Zhao, R., Jumaa, K. B., et al.: Anthracene Photolysis in Aqueous Solution and Ice: Photon Flux Dependence and Comparison of Kinetics in Bulk Ice and at the Air-Ice Interface, Environ. Sci. Technol., in press, 2010.

Klan, P. and Holoubek, I.: Ice (photo)chemistry. Ice as a medium for long-term (photo)chemical transformations - environmental implications, Chemosphere, 46, 1201-1210, 2002. 
Klan, P., Klanova, J., Holoubeket, I., et al.: Photochemical activity of organic compounds in ice induced by sunlight irradiation: The Svalbard project." Geophys. Res. Lett., 30, 46-1-46-4, 2003.

Klanova, J., Klan, P., Heger, D., et al.: Comparison of the effects of $\mathrm{UV}, \mathrm{H}_{2} \mathrm{O}_{2} / \mathrm{UV}$ and $\gamma \alpha \mu \mu \alpha$-irradiation processes on frozen and liquid water solutions of monochlorophenols, Photochem. Photobiol. Sci., 2, 1023-1031, 2003a.

Klanova, J., Klan, P., Nosek, J., et al.: Environmental ice photochemistry: Monochlorophenols, Environ. Sci. Technol., 37, 1568-1574, 2003b.

Lee, C. W., Lee, P. R., Kim, Y. K., et al.: Mechanistic study of proton transfer and H/D exchange in ice films at low temperatures (100-140 K), J. Chem. Phys., 127, 084701, doi:10.1063/1.2759917, 2007.

Masclet, P., Hoyau, V., Jaffrezo, J. L., et al.: Polycyclic aromatic hydrocarbon deposition on the ice sheet of Greenland. Part I: Superficial snow, Atmos. Environ., 34(19), 3195-3207, 2000.

Matykiewiczova, N., Kurkova, R., Klanova, J., et al.: Photochemically induced nitration and hydroxylation of organic aromatic compounds in the presence of nitrate or nitrite in ice, J. Photochem. Photobiol., 187(1), 24-32, 2007.

Melnikov, S., Carroll, J., Gorshkov, A., et al.: Snow and ice concentrations of selected persistent pollutants in the Ob-Yenisey River watershed, Sci. Total Environ., 306(1-3), 27-37, 2003.

Mmereki, B. T. and Donaldson, D. J.: Direct observation of the kinetics of an atmospherically important reaction at the air-aqueous interface, J. Phys. Chem. A, 107, 11038-11042, 2003.

Pan, X., Bass, A. D., Jay-Gerin, J.-P., and Sanche, L.: A mechanism for the production of hydrogen peroxide and the hydroperoxyl radical on icy satellites by low-energy electrons, Icarus, 172, 521-525, 2004.

Qiu, R., Green, S. A., Honrath, R. E., et al.: Measurements of $J\left(\mathrm{NO}_{3}\right)(-)$ in snow by nitrate-based actinometry, Atmos. Environ., 36(15-16), 2563-2571, 2002.

Ram, K. and Anastasio, C.: Photochemistry of phenanthrene, pyrene, and fluoranthene in ice and snow, Atmos. Environ., 43, 2252-2259, 2009.
Schrimpff, E., Thomas, W., Herrmann, R., et al.: Regional Patterns of Contaminants (Pah, Pesticides and Trace-Metals) in Snow of Northeast Bavaria and Their Relationship to Human Influence and Orographic Effects, Water Air Soil Poll., 11(4), 481-497, 1979.

Slater, J. F., Currie, L. A., Dibb, J. E., et al.: Distinguishing the relative contribution of fossil fuel and biomass combustion aerosols deposited at Summit, Greenland through isotopic and molecular characterization of insoluble carbon, Atmos. Environ., 36(28), 4463-4477, 2002.

Stella, L., Seraglia, R., Sturaro, A., et al.: On the photochemical oxidation of benzene and its relevance at environmental level, Rapid Communications in Mass Spectrometry, 22(2), 257-260, 2008.

Tubaro, M., Marotta, E., Seraglia, R., et al.: Atmospheric pressure photoionization mechanisms. 2. The case of benzene and toluene, Rapid Comm. Mass Spectrom., 17(21), 2423-2429, 2003.

Vione, D., Falletti, G., Maurino, V., et al.: Sources and sinks of hydroxyl radicals upon irradiation of natural water samples, Environ. Sci. Technol., 40(12), 3775-3781, 2006.

Wang, X. P., Xu, B. Q., Kang, S. C., et al.: The historical residue trends of DDT, hexachlorocyclohexanes and polycyclic aromatic hydrocarbons in an ice core from Mt. Everest, central Himalayas, China, Atmos. Environ., 42(27), 6699-6709, 2008 a.

Wang, X. P., T. D. Yao, et al. (2008b). "The recent deposition of persistent organic pollutants and mercury to the Dasuopu glacier, Mt. Xixiabangma, central Himalayas, Sci. Total Environ., 394(1), 134-143.

Zheng, W., Jewitt, D., and Kaiser, R. I.: Formation of hydrogen, oxygen, and hydrogen peroxide in electron-irradiated crystalline water ice, The Astrophysical Journal, 639, 534-548, 2006. 